\title{
Socio-economic Status and Associated Factors in the Uptake of HIV Testing: Findings from the South African Population-based National Household Survey Conducted in 2017
}

Sean Jooste ( $\nabla$ sjooste@hsrc.ac.za )

Human Sciences Research Council https://orcid.org/0000-0002-3984-5997

Musawenkosi Mabaso

Human Sciences Research Council

Myra Taylor

University of KwaZulu-Natal School of Nursing and Public Health

Alicia North

Human Sciences Research Council

Yolande Shean

Human Sciences Research Council

Leickness Chisamu Simbayi

Human Sciences Research Council

Research article

Keywords: UNAIDS 90-90-90 targets, HIV testing, Socio-economic status, South Africa

Posted Date: November 18th, 2020

DOI: https://doi.org/10.21203/rs.3.rs-108686/v1

License: (a) (i) This work is licensed under a Creative Commons Attribution 4.0 International License. Read Full License 


\section{Abstract}

Background: Improved understanding of barriers to HIV testing is important for reaching the first of the UNAIDS 90-90-90 targets, which states that 90 percent of HIV positive individuals ought to know their HIV status. This study examined socioeconomic status (SES) factors and related covariates associated with HIV testing uptake in the general population in South Africa. Information on SES and HIV testing may impact the HIV treatment and care cascade and prevention of HIV transmission.

Methods: This study used data obtained from a national cross-sectional, population-based household survey conducted in 2017 using a multi-stage stratified random cluster sampling design. A composite SES score was created using multiple correspondence analyses of household assets; households were classified into wealth quintiles and dichotomized into low SES/poorest (lowest 3 quintiles) and high SES/less-poor (highest 2 quintiles). Bivariate and multivariate logistic regression models were fitted to analyse associations between the uptake of HIV testing and a set of socio-demographic and HIV-related variables.

Results: HIV testing uptake was $73.8 \%$ and $76.7 \%$ among low and high SES household, respectively, both of which were below the first 90 target. The findings showed increased uptake of HIV testing was associated with being female and having a higher educational level independent of SES. Decreased uptake of HIV testing was associated with being from White and Indian/Asian race groups, residing in rural formal areas, alcohol consumption, and high self-perceived risk of HIV infection.

Conclusions: We found that HIV testing uptake was similar for people from both low and high SES. However, interventions aimed at improving HIV testing in order to reach the first 90 target, should target males, all race groups, those with low education attainment, and those residing in rural formal areas. Reaching the first target will require efforts to improve community knowledge, and awareness about the negative impact of alcohol use and address HIV risk perception.

\section{Background}

Sub-Saharan Africa (SSA) bears the largest burden of the HIV epidemic with $53 \%$ of the world's 36.9 million [31.1-43.9 million] people living with HIV in 2018 [1]. The HIV epidemic in South Africa is the largest globally, with a national prevalence of $14 \%$, which translated to an estimated 7.9 million people living with HIV in 2017 [2]. HIV testing uptake serves as the foundation for the Joint United Nations Programme on HIV/AIDS (UNAIDS) strategic framework in the fight against HIV [3]. This framework specifies that $90 \%$ of HIV-positive people should be aware of their status, $90 \%$ of those diagnosed should receive sustained antiretroviral therapy (ART), and that $90 \%$ of those on ART achieve viral suppression [3]. This framework was adopted in December 2014 by the South African government as the basis of its national response to the HIV/AIDS epidemic in the country. Achieving the UNAIDS and South African government targets hinges on reaching the key goal of identifying $90 \%$ of people living with HIV.

HIV testing is crucial as it provides a diagnosis for people living with HIV for linkage to care and treatment [4]. Globally, progress is being made, and at the end of 2017, three-quarters of people living with HIV knew their status [1]. Despite the excellent strides made with HIV testing in SSA, awareness of HIV status remains lower 
than the rest of the world. Findings from 10 population-based studies conducted in 2012, identified that the average percentage of people living with HIV who know their status was below $40 \%$ [5].

In Southern Africa, despite the progress South Africa has towards reaching the 90-90-90 goals, there are still barriers to HIV testing [3]. Several barriers to HIV testing have been identified in studies that have been done in SSA. In the 2005 Demographic and Health Survey in Cote d'Ivoire, a low socio-economic status was related to lower proportions of individuals testing for HIV testing, which included lack of or lower educational attainment and employment status [6, 7]. Furthermore, cross-sectional surveys conducted as part of the Multi-country African Testing and Counselling for HIV (MATCH) study in Burkina Faso, Kenya, Malawi, and Uganda found that a higher SES was an indicator of the probability of HIV testing [8].

Other barriers identified from various studies included a perceived low risk of infection $[5,7,9,10]$ as well as fear of finding out ones' status and perceived stigma [10-13]. A cross-sectional study in 2011 in Ethiopia found that HIV testing was lower in rural communities with lower SES [14]. A household survey conducted in 2007 in ten countries in Southern Africa found that people living in urban areas are more likely to be tested [15].

Knowing one's HIV status should be independent of one's SES and where one resides [16]. Unless these barriers to testing are addressed, the final goal of the UNAIDS targets where $90 \%$ of people who are on treatment are virally suppressed will not be attained [3]. Improved understanding of testing barriers is important to allow interventions focused on reaching the first 90 target. This study examined SES factors and related covariates associated with self-reported history of ever testing for HIV using a national survey in South Africa.

\section{Methods}

\section{Data}

This analysis used data from a national cross-sectional, population-based household survey "South African national HIV prevalence, incidence, behaviour and communication survey" conducted in 2017 using a multistage stratified random cluster sampling approach, described in detail in the survey report [2]. In summary, a total of 1000 small area layers (SALs) were sampled using the 2015 national population sampling frame of 103000 SALs developed by Statistics South Africa [17]. The selection of SALs was stratified by province, locality type (urban formal, rural formal, and rural informal/tribal areas) and race groups in urban areas. A total of 15 visiting points (VPs) were randomly selected from each of the 1000 SALs, targeting 15000 VPs. Of these, 12435 (82.9\%) VPs were approached. Among these VPs, 11776 (94.7\%) were valid and a household response rate of $82.2 \%$ was achieved from the valid VPs. All members of the selected households were eligible to participate in the survey.

The survey collected data using a household questionnaire and three age-appropriate questionnaires were administered to consenting individuals. For those younger than 18 years of age, consent was given by parents/guardians and assent by the participant. The interview instruments solicited information among others on socio-demographic characteristics, HIV-related knowledge, attitudes, and behaviours, including questions on HIV testing. Questionnaire data were collected digitally using electronic tablets. Fieldworkers also collected dried blood specimen samples from participants using a finger prick. Samples were sent to a centralised 
laboratory for HIV antibodies. The focus of the present study was on youth and adults 15 years and older who responded to the question on HIV testing.

\section{Primary outcome variable}

The dependent primary outcome variable 'HIV testing' was ascertained by the question: "Have you ever been tested for HIV? Yes=1 and No=0." Ever been tested was defined as having accessed HIV testing services at least once before the survey.

\section{Explanatory variables}

Independent variables included socio-demographic characteristics and socio-economic characteristics. Sociodemographic characteristics comprised age (15 to 24 years, 25 to 49 years, and 50+ years) sex (male and female), race (Black African, White, Coloured, and Indian/Asian), and current marital status (married and not married; which included divorced/separated and widowed/widow). Socio-economic characteristics included highest educational level completed (no education, primary, secondary, and tertiary), employment status (not employed and employed), locality type (urban formal, rural informal, rural formal), and asset-based SES which was constructed using multiple correspondence analyses (MCA), based on questions on the availability of essential services and ownership of a range of household assets [18]. MCA is a data reduction technique for categorical data, which calculates a composite indicator score computed by adding up all the weighted responses. The predicted score for each household was used to compute five quintiles (1st lowest, 2nd lower, 3rd middle, 4th higher and 5th highest) representing a continuum of household SES from the poorest to the least poor. The quintiles were then dichotomized into low SES or poorest (lowest 3 quintiles) high SES or lesspoor (highest 2 quintiles).

HIV-related risk factors included age at sexual debut (less than 15 years and more than 15 years), age-disparate partnerships (partner older by 5 years, partner younger by 5 years, a partner within 5 years), multiple sexual partners in the last 12 months (one partner, and two or more sexual partners), condom use at last sex (no and yes), alcohol use risk score (abstainers, low, high, and hazardous risk drinkers) based on the Alcohol Use Disorder Identification Test (AUDIT) scale [19, 20], correct HIV knowledge and myth rejection (yes and no) based on responses from the following questions (Can AIDS be cured? Can a person reduce the risk of HIV by having fewer sexual partners? Can a healthy-looking person have HIV? Can a person get HIV by sharing food with someone who is infected? Can a person reduce the risk of getting HIV by using a condom every time he/she has sex?), self-perceived risk of HIV infection (no and yes).

\section{Statistical analysis}

All statistical analysis was done in STATA 15.0 (Stata Corporation, College Station, Texas, USA) software. The 'svy'command was used to introduce weights which take into account the complex design of the survey. Data descriptive statistics (unweighted frequencies and weighted percentages) were used to summarize sociodemographic characteristics and HIV-related risk factors of the study sample. The Pearson's chi-square test was used compare differences in proportions between categorical variables. Bivariate logistic regression was used 
to assess the relationship between HIV testing uptake and the explanatory variable. Statistically significant variables were entered into a multivariate logistic regression model to examine the independent effects of covariates associated with the uptake of HIV testing. The analysis was stratified by asset-based SES (low and high). Crude and adjusted odds ratios (aORs) with 95\% confidence intervals (Cl) , and p-values less than 0.05 were reported for all statistically significant associations. Coefficient plots were used to display the results of the final models [21].

\section{Results}

\section{Background characteristics of the study sample}

Table 1 shows that over half of the sample was aged $25-49$ years (53.5\%), female (52.1\%), Black African (79.0\%), not married (70.8\%), had completed secondary education (67.6\%), and resided in urban areas (69.5\%). There were significant differences in characteristics between participants with low and high socio-economic status with regards to age, sex, race, marital status, level of education, employment status, and locality type ( $p$ $<0.001)$. 
Table 1

Background characteristics of the sample by SES among 15 years and older, South Africa 2017

\begin{tabular}{|c|c|c|c|c|c|c|c|}
\hline \multirow[t]{2}{*}{ Variable } & \multicolumn{2}{|c|}{ Overall sample } & \multicolumn{2}{|c|}{ Low SES } & \multicolumn{2}{|c|}{ High SES } & \multirow[b]{2}{*}{$p$-values } \\
\hline & $\mathrm{n}$ & $\% *$ & $\mathrm{n}$ & $\%$ & $\mathrm{n}$ & $\%$ & \\
\hline Overall sample & 21075 & 100 & 8504 & 100 & 12571 & 100 & \\
\hline \multicolumn{8}{|l|}{ Age categories } \\
\hline $15-19$ years & 2762 & 11.5 & 1242 & 12.3 & 1520 & 10.8 & $<0.001$ \\
\hline $20-24$ years & 2578 & 12.4 & 1209 & 14.3 & 1369 & 11.0 & \\
\hline $25-49$ years & 9715 & 53.5 & 4021 & 54.9 & 5694 & 52.4 & \\
\hline $50+$ years & 6020 & 22.6 & 2032 & 18.6 & 3988 & 25.8 & \\
\hline \multicolumn{8}{|l|}{ Sex } \\
\hline Male & 8812 & 47.9 & 3556 & 47.6 & 5256 & 48.2 & 0.481 \\
\hline Female & 12263 & 52.1 & 4948 & 52.4 & 7315 & 51.8 & \\
\hline \multicolumn{8}{|l|}{ Race } \\
\hline Black African & 13747 & 79.0 & 7551 & 95.2 & 6196 & 66.1 & $<0.001$ \\
\hline White & 1509 & 9.3 & 24 & 0.4 & 1485 & 16.3 & \\
\hline Coloured & 3805 & 8.9 & 875 & 4.2 & 2930 & 12.6 & \\
\hline Indian/Asian & 2014 & 2.9 & 54 & 0.2 & 1960 & 5.0 & \\
\hline \multicolumn{8}{|l|}{ Current marital status } \\
\hline Married & 6758 & 29.2 & 1751 & 19.6 & 5007 & 36.8 & $<0.001$ \\
\hline Not married & 14312 & 70.8 & 6752 & 80.4 & 7560 & 63.2 & \\
\hline \multicolumn{8}{|c|}{ Highest educational level obtained } \\
\hline No education/primary & 3278 & 16.8 & 1874 & 24.9 & 1404 & 10.9 & $<0.001$ \\
\hline Secondary & 10263 & 67.6 & 3889 & 70.4 & 6374 & 65.5 & \\
\hline Tertiary & 2276 & 15.6 & 201 & 4.7 & 2075 & 23.6 & \\
\hline \multicolumn{8}{|l|}{ Employment status } \\
\hline Unemployed & 13432 & 63.8 & 6053 & 71.7 & 7379 & 57.6 & $<0.001$ \\
\hline Employed & 7352 & 36.2 & 2355 & 28.3 & 4997 & 42.4 & \\
\hline \multicolumn{8}{|l|}{ Locality type } \\
\hline Urban & 13810 & 69.5 & 3202 & 48.5 & 10608 & 86.2 & $<0.001$ \\
\hline
\end{tabular}




\begin{tabular}{|lccccccc|}
\hline Variable & Overall sample & \multicolumn{3}{c|}{ Low SES } & \multicolumn{3}{c|}{ High SES } \\
\hline Rural informal (tribal areas) & 4909 & 25.8 & 3650 & 44.8 & 1259 & 10.8 \\
\hline Rural (farms) & 2356 & 4.7 & 1652 & 6.7 & 704 & 3.1 \\
\hline *Weighted percentages & & & & & & \\
\hline
\end{tabular}

Table 2 shows the reported uptake of HIV testing among the population aged 15 years and older, by assetbased socio-economic status and socio-demographic characteristics of the study sample. Overall, people with a high SES reported significantly higher HIV testing uptake than those with a low SES, 76.7\% vs 73.8\% $(p<0.001)$. There were significant differences in HIV testing by SES among people aged 25-49 years, males, Black African, employed, and those residing in rural informal areas (all $p<0.001$ ). HIV testing uptake for those aged 50 years and older, not married, residing in urban and rural formal areas were also significantly different between low and high SES (all $p<0.05$ ). 
Table 2

HIV testing socio-demographic characteristics by SES among 15 years and older, South Africa 2017

\begin{tabular}{|c|c|c|c|c|c|c|c|c|c|c|}
\hline \multirow[t]{2}{*}{ Variable } & \multicolumn{3}{|c|}{ Total } & \multicolumn{3}{|c|}{ Low SES } & \multicolumn{4}{|c|}{ High SES } \\
\hline & $\mathrm{n}$ & Tested & $\begin{array}{l}95 \% \\
\mathrm{Cl}\end{array}$ & $\mathrm{n}$ & Tested & $\begin{array}{l}95 \% \\
\mathrm{Cl}\end{array}$ & $\mathrm{n}$ & Tested & $\begin{array}{l}95 \% \\
\mathrm{Cl}\end{array}$ & $\begin{array}{l}\mathrm{p}- \\
\text { value }\end{array}$ \\
\hline Overall & $\begin{array}{l}21 \\
075\end{array}$ & 75.4 & $\begin{array}{l}74.2- \\
76.6\end{array}$ & $\begin{array}{l}8 \\
504\end{array}$ & 73.8 & $\begin{array}{l}72.0- \\
75.6\end{array}$ & $\begin{array}{l}12 \\
571\end{array}$ & 76.7 & $\begin{array}{l}75.3- \\
78.1\end{array}$ & $\begin{array}{l}< \\
0.001\end{array}$ \\
\hline \multicolumn{11}{|c|}{ Age categories } \\
\hline $15-19$ years & $\begin{array}{l}2 \\
762\end{array}$ & 41.9 & $\begin{array}{l}38.9- \\
45.0\end{array}$ & $\begin{array}{l}1 \\
242\end{array}$ & 42.0 & $\begin{array}{l}37.7- \\
46.4\end{array}$ & $\begin{array}{l}1 \\
520\end{array}$ & 41.9 & $\begin{array}{l}38.2- \\
45.7\end{array}$ & 0.958 \\
\hline $20-24$ years & $\begin{array}{l}2 \\
578\end{array}$ & 74.1 & $\begin{array}{l}71.6- \\
76.5\end{array}$ & $\begin{array}{l}1 \\
209\end{array}$ & 73.2 & $\begin{array}{l}69.9- \\
76.2\end{array}$ & $\begin{array}{l}1 \\
369\end{array}$ & 75.1 & $\begin{array}{l}71.6- \\
78.4\end{array}$ & 0.271 \\
\hline $25-49$ years & $\begin{array}{l}9 \\
715\end{array}$ & 85.2 & $\begin{array}{l}83.8- \\
86.6\end{array}$ & $\begin{array}{l}4 \\
021\end{array}$ & 83.0 & $\begin{array}{l}80.8- \\
85.0\end{array}$ & $\begin{array}{l}5 \\
694\end{array}$ & 87.1 & $\begin{array}{l}85.4- \\
88.6\end{array}$ & $\hat{0}_{0.001}^{<}$ \\
\hline $50+$ years & $\begin{array}{l}6 \\
020\end{array}$ & 69.9 & $\begin{array}{l}68.1- \\
71.6\end{array}$ & $\begin{array}{l}2 \\
032\end{array}$ & 68.2 & $\begin{array}{l}65.4- \\
70.8\end{array}$ & $\begin{array}{l}3 \\
988\end{array}$ & 70.9 & $\begin{array}{l}68.6- \\
73.1\end{array}$ & 0.031 \\
\hline \multicolumn{11}{|c|}{ Sex of respondent } \\
\hline Male & $\begin{array}{l}8 \\
812\end{array}$ & 71.2 & $\begin{array}{l}69.4- \\
72.8\end{array}$ & $\begin{array}{l}3 \\
556\end{array}$ & 67.3 & $\begin{array}{l}64.5- \\
69.9\end{array}$ & $\begin{array}{l}5 \\
256\end{array}$ & 74.2 & $\begin{array}{l}72.2- \\
76.1\end{array}$ & $\stackrel{<}{0.001}$ \\
\hline Female & $\begin{array}{l}12 \\
263\end{array}$ & 79.4 & $\begin{array}{l}78.0- \\
80.6\end{array}$ & $\begin{array}{l}4 \\
948\end{array}$ & 79.8 & $\begin{array}{l}77.8- \\
81.6\end{array}$ & $\begin{array}{l}7 \\
315\end{array}$ & 79.0 & $\begin{array}{l}77.4- \\
80.6\end{array}$ & 0.283 \\
\hline \multicolumn{11}{|l|}{ Race } \\
\hline African & $\begin{array}{l}13 \\
747\end{array}$ & 76.7 & $\begin{array}{l}75.2- \\
78.1\end{array}$ & $\begin{array}{l}7 \\
551\end{array}$ & 74.0 & $\begin{array}{l}72.1- \\
75.8\end{array}$ & $\begin{array}{l}6 \\
196\end{array}$ & 79.7 & $\begin{array}{l}77.9- \\
81.4\end{array}$ & $\stackrel{<}{0.001}$ \\
\hline White & $\begin{array}{l}1 \\
509\end{array}$ & 69.7 & $\begin{array}{l}66.0- \\
73.2\end{array}$ & 24 & 64.5 & $\begin{array}{l}43.5- \\
81.1\end{array}$ & $\begin{array}{l}1 \\
485\end{array}$ & 69.8 & $\begin{array}{l}66.1- \\
73.3\end{array}$ & 0.575 \\
\hline Coloured & $\begin{array}{l}3 \\
805\end{array}$ & 74.1 & $\begin{array}{l}72.0- \\
76.1\end{array}$ & 875 & 71.6 & $\begin{array}{l}65.6- \\
76.9\end{array}$ & $\begin{array}{l}2 \\
930\end{array}$ & 74.7 & $\begin{array}{l}72.6- \\
76.7\end{array}$ & 0.067 \\
\hline Indian/A & $\begin{array}{l}2 \\
014\end{array}$ & 63.9 & $\begin{array}{l}60.1- \\
67.6\end{array}$ & 54 & 62.7 & $\begin{array}{l}41.9- \\
79.7\end{array}$ & $\begin{array}{l}1 \\
960\end{array}$ & 64.0 & $\begin{array}{l}60.1- \\
67.7\end{array}$ & 0.844 \\
\hline \multicolumn{11}{|c|}{$\begin{array}{l}\text { Current marital } \\
\text { status }\end{array}$} \\
\hline Married & $\begin{array}{l}6 \\
758\end{array}$ & 81.5 & $\begin{array}{l}79.8- \\
83.0\end{array}$ & $\begin{array}{l}1 \\
751\end{array}$ & 80.5 & $\begin{array}{l}77.5- \\
83.2\end{array}$ & $\begin{array}{l}5 \\
007\end{array}$ & 81.9 & $\begin{array}{l}79.9- \\
83.7\end{array}$ & 0.194 \\
\hline Not married & $\begin{array}{l}14 \\
312\end{array}$ & 72.9 & $\begin{array}{l}71.5- \\
74.3\end{array}$ & $\begin{array}{l}6 \\
752\end{array}$ & 72.2 & $\begin{array}{l}70.2- \\
74.2\end{array}$ & $\begin{array}{l}7 \\
560\end{array}$ & 73.7 & $\begin{array}{l}71.9- \\
75.3\end{array}$ & 0.044 \\
\hline $\begin{array}{l}\text { Highest level o } \\
\text { education } \\
\text { obtained }\end{array}$ & & & & & & & & & & \\
\hline
\end{tabular}




\begin{tabular}{|c|c|c|c|c|c|c|c|c|c|c|}
\hline \multirow{2}{*}{$\begin{array}{l}\text { Variable } \\
\text { No } \\
\text { education/primary }\end{array}$} & \multicolumn{3}{|l|}{ Total } & \multicolumn{3}{|c|}{ Low SES } & \multicolumn{4}{|c|}{ High SES } \\
\hline & $\begin{array}{l}3 \\
278\end{array}$ & 71.9 & $\begin{array}{l}69.6- \\
74.1\end{array}$ & $\begin{array}{l}1 \\
874\end{array}$ & 72.6 & $\begin{array}{l}69.4- \\
75.6\end{array}$ & $\begin{array}{l}1 \\
404\end{array}$ & 70.7 & $\begin{array}{l}67.2- \\
74.0\end{array}$ & 0.232 \\
\hline Secondary & $\begin{array}{l}10 \\
263\end{array}$ & 80.4 & $\begin{array}{l}79.0- \\
81.8\end{array}$ & $\begin{array}{l}3 \\
889\end{array}$ & 81.0 & $\begin{array}{l}79.0- \\
82.8\end{array}$ & $\begin{array}{l}6 \\
374\end{array}$ & 80.0 & $\begin{array}{l}78.0- \\
81.8\end{array}$ & 0.216 \\
\hline Tertiary & $\begin{array}{l}2 \\
276\end{array}$ & 86.1 & $\begin{array}{l}83.7- \\
88.2\end{array}$ & 201 & 89.1 & $\begin{array}{l}82.9- \\
93.2\end{array}$ & $\begin{array}{l}2 \\
075\end{array}$ & 85.7 & $\begin{array}{l}83.2- \\
87.9\end{array}$ & 0.185 \\
\hline \multicolumn{11}{|l|}{$\begin{array}{l}\text { Employment } \\
\text { status }\end{array}$} \\
\hline Unemployed & $\begin{array}{l}13 \\
432\end{array}$ & 70.7 & $\begin{array}{l}69.3- \\
72.2\end{array}$ & $\begin{array}{l}6 \\
053\end{array}$ & 71.0 & $\begin{array}{l}69.0- \\
72.9\end{array}$ & $\begin{array}{l}7 \\
379\end{array}$ & 70.5 & $\begin{array}{l}68.6- \\
72.3\end{array}$ & 0.526 \\
\hline Employed & $\begin{array}{l}7 \\
352\end{array}$ & 84.0 & $\begin{array}{l}82.3- \\
85.5\end{array}$ & $\begin{array}{l}2 \\
355\end{array}$ & 81.3 & $\begin{array}{l}78.3- \\
83.9\end{array}$ & $\begin{array}{l}4 \\
997\end{array}$ & 85.5 & $\begin{array}{l}83.6- \\
87.2\end{array}$ & $\begin{array}{l}< \\
0.001\end{array}$ \\
\hline \multicolumn{11}{|l|}{ Locality type } \\
\hline Urban areas & $\begin{array}{l}13 \\
810\end{array}$ & 77.5 & $\begin{array}{l}76.1- \\
78.9\end{array}$ & $\begin{array}{l}3 \\
202\end{array}$ & 78.7 & $\begin{array}{l}76.1- \\
81.0\end{array}$ & $\begin{array}{l}10 \\
608\end{array}$ & 77.0 & $\begin{array}{l}75.5- \\
78.5\end{array}$ & 0.044 \\
\hline $\begin{array}{l}\text { Rural informal } \\
\text { areas }\end{array}$ & $\begin{array}{l}4 \\
909\end{array}$ & 70.5 & $\begin{array}{l}68.2- \\
72.8\end{array}$ & $\begin{array}{l}3 \\
650\end{array}$ & 69.3 & $\begin{array}{l}66.7- \\
71.7\end{array}$ & $\begin{array}{l}1 \\
259\end{array}$ & 74.7 & $\begin{array}{l}70.6- \\
78.4\end{array}$ & $\dot{0}_{0.001}$ \\
\hline Rural formal areas & $\begin{array}{l}2 \\
356\end{array}$ & 71.1 & $\begin{array}{l}67.0- \\
75.0\end{array}$ & $\begin{array}{l}1 \\
652\end{array}$ & 69.4 & $\begin{array}{l}64.0- \\
74.4\end{array}$ & 704 & 74.0 & $\begin{array}{l}68.2- \\
79.1\end{array}$ & 0.025 \\
\hline
\end{tabular}

Table 3 shows the reported uptake of HIV testing among the population aged 15 years and older by asset-based socio-economic status by HIV-related risk characteristics of the study sample. There were significant differences found in reported HIV testing between the people from low and high SES with for those with no self-perceived HIV risk and survey HIV status result (both $p<0.001$ ). Differences were also found among people that ever had sex and those who did not have sex, differences with people that have a sexual partner five years and older than themselves, those that do not drink alcohol and risky alcohol drinkers, and correct knowledge of HIV prevention and transmission (all $\mathrm{p}<0.05$ ). 
Table 3

HIV testing related risk characteristics by SES among 15 years and older, South Africa 2017

\begin{tabular}{|c|c|c|c|c|c|c|c|c|c|c|}
\hline \multirow{2}{*}{$\begin{array}{l}\text { Variable } \\
\text { Sexual activity }\end{array}$} & \multicolumn{3}{|l|}{ Total } & \multicolumn{3}{|c|}{ Low SES } & \multicolumn{4}{|c|}{ High SES } \\
\hline & $\mathrm{n}$ & Tested & $\begin{array}{l}95 \% \\
\mathrm{Cl}\end{array}$ & $\mathrm{N}$ & Tested & $\begin{array}{l}95 \% \\
\mathrm{Cl}\end{array}$ & $\mathrm{n}$ & Tested & $\begin{array}{l}95 \% \\
\mathrm{Cl}\end{array}$ & $\begin{array}{l}\mathrm{p}- \\
\text { value }\end{array}$ \\
\hline Never had sex & $\begin{array}{l}3 \\
142\end{array}$ & 42.3 & $\begin{array}{l}39.3- \\
45.3\end{array}$ & $\begin{array}{l}1 \\
368\end{array}$ & 41.0 & $\begin{array}{l}37.0- \\
45.1\end{array}$ & $\begin{array}{l}1 \\
920\end{array}$ & 45.1 & $\begin{array}{l}41.3- \\
49.0\end{array}$ & 0.019 \\
\hline Had sex & $\begin{array}{l}15 \\
741\end{array}$ & 81.2 & $\begin{array}{l}80.0- \\
82.3\end{array}$ & $\begin{array}{l}6 \\
766\end{array}$ & 80.4 & $\begin{array}{l}78.8- \\
81.9\end{array}$ & $\begin{array}{l}9 \\
895\end{array}$ & 82.1 & $\begin{array}{l}80.5- \\
83.5\end{array}$ & 0.006 \\
\hline \multicolumn{11}{|l|}{ Sexual debut } \\
\hline $\begin{array}{l}\text { Sex before the } \\
\text { age of } 15 \text { years }\end{array}$ & 324 & 67.4 & $\begin{array}{l}60.4- \\
73.7\end{array}$ & 169 & 65.6 & $\begin{array}{l}56.6- \\
73.5\end{array}$ & 173 & 69.5 & $\begin{array}{l}59.0- \\
78.3\end{array}$ & 0.441 \\
\hline $\begin{array}{l}\text { Sex at } 15 \text { years } \\
\text { and older }\end{array}$ & $\begin{array}{l}4 \\
859\end{array}$ & 57.2 & $\begin{array}{l}54.8- \\
59.6\end{array}$ & $\begin{array}{l}2 \\
269\end{array}$ & 58.0 & $\begin{array}{l}54.7- \\
61.2\end{array}$ & $\begin{array}{l}2 \\
710\end{array}$ & 57.7 & $\begin{array}{l}54.6- \\
60.7\end{array}$ & 0.831 \\
\hline \multicolumn{11}{|l|}{$\begin{array}{l}\text { Age of sexual } \\
\text { partner }\end{array}$} \\
\hline $\begin{array}{l}\text { Partner more } \\
\text { than } 5 \text { years } \\
\text { younger }\end{array}$ & $\begin{array}{l}1 \\
971\end{array}$ & 82.2 & $\begin{array}{l}79.6- \\
84.5\end{array}$ & $\begin{array}{l}2 \\
344\end{array}$ & 82.3 & $\begin{array}{l}80.0- \\
84.4\end{array}$ & $\begin{array}{l}3 \\
908\end{array}$ & 85.2 & $\begin{array}{l}83.2- \\
87.0\end{array}$ & 0.002 \\
\hline $\begin{array}{l}\text { Partner within } \\
\text { five years }\end{array}$ & $\begin{array}{l}5 \\
849\end{array}$ & 84.3 & $\begin{array}{l}82.7- \\
85.8\end{array}$ & 813 & 81.3 & $\begin{array}{l}77.2- \\
84.8\end{array}$ & $\begin{array}{l}1 \\
179\end{array}$ & 83.2 & $\begin{array}{l}79.8- \\
86.1\end{array}$ & 0.274 \\
\hline $\begin{array}{l}\text { Partner more } \\
\text { than } 5 \text { years } \\
\text { older }\end{array}$ & $\begin{array}{l}2 \\
308\end{array}$ & 89.8 & $\begin{array}{l}88.1- \\
91.3\end{array}$ & $\begin{array}{l}1 \\
073\end{array}$ & 90.3 & $\begin{array}{l}87.7- \\
92.4\end{array}$ & $\begin{array}{l}1 \\
414\end{array}$ & 90.0 & $\begin{array}{l}87.6- \\
92.0\end{array}$ & 0.804 \\
\hline \multicolumn{11}{|c|}{ Number of sexual partners in the past 12 months } \\
\hline 1 sexual partner & $\begin{array}{l}9 \\
289\end{array}$ & 85.2 & $\begin{array}{l}84.0- \\
86.4\end{array}$ & $\begin{array}{l}3 \\
827\end{array}$ & 84.5 & $\begin{array}{l}82.7- \\
86.1\end{array}$ & $\begin{array}{l}6 \\
024\end{array}$ & 85.7 & $\begin{array}{l}84.1- \\
87.2\end{array}$ & 0.102 \\
\hline $\begin{array}{l}2+\text { or more } \\
\text { sexual partners }\end{array}$ & 892 & 82.5 & $\begin{array}{l}78.8- \\
85.6\end{array}$ & 438 & 79.4 & $\begin{array}{l}74.2- \\
83.8\end{array}$ & 504 & 86.2 & $\begin{array}{l}81.5- \\
89.9\end{array}$ & 0.006 \\
\hline \multicolumn{11}{|c|}{ Condom use at last sex in the past 12 months } \\
\hline No condom use & $\begin{array}{l}6 \\
564\end{array}$ & 84.9 & $\begin{array}{l}83.4- \\
86.2\end{array}$ & $\begin{array}{l}2 \\
445\end{array}$ & 83.8 & $\begin{array}{l}81.3- \\
86.1\end{array}$ & $\begin{array}{l}4 \\
535\end{array}$ & 85.4 & $\begin{array}{l}83.7- \\
87.0\end{array}$ & 0.075 \\
\hline Yes condom use & $\begin{array}{l}3 \\
541\end{array}$ & 85.0 & $\begin{array}{l}83.3- \\
86.6\end{array}$ & $\begin{array}{l}1 \\
817\end{array}$ & 84.1 & $\begin{array}{l}81.7- \\
86.1\end{array}$ & $\begin{array}{l}1 \\
919\end{array}$ & 86.3 & $\begin{array}{l}84.0- \\
88.3\end{array}$ & 0.058 \\
\hline \multicolumn{11}{|l|}{ AUDIT Score } \\
\hline Abstainers & $\begin{array}{l}13 \\
037\end{array}$ & 73.5 & $\begin{array}{l}71.9- \\
75.1\end{array}$ & $\begin{array}{l}5 \\
974\end{array}$ & 72.9 & $\begin{array}{l}70.7- \\
75.0\end{array}$ & $\begin{array}{l}7 \\
835\end{array}$ & 74.7 & $\begin{array}{l}72.9- \\
76.5\end{array}$ & 0.017 \\
\hline Low risk (1-7) & $\begin{array}{l}3 \\
537\end{array}$ & 77.9 & $\begin{array}{l}75.6- \\
80.0\end{array}$ & $\begin{array}{l}1 \\
177\end{array}$ & 76.8 & $\begin{array}{l}73.5- \\
79.8\end{array}$ & $\begin{array}{l}2 \\
532\end{array}$ & 78.7 & $\begin{array}{l}75.7- \\
81.4\end{array}$ & 0.193 \\
\hline
\end{tabular}




\begin{tabular}{|c|c|c|c|c|c|c|c|c|c|c|}
\hline \multirow{2}{*}{$\begin{array}{l}\text { Variable } \\
\begin{array}{l}\text { Risky level (8- } \\
15)\end{array}\end{array}$} & \multicolumn{3}{|c|}{ Total } & \multicolumn{3}{|c|}{ Low SES } & \multicolumn{4}{|c|}{ High SES } \\
\hline & $\begin{array}{l}1 \\
257\end{array}$ & 79.2 & $\begin{array}{l}75.5- \\
82.4\end{array}$ & 557 & 76.2 & $\begin{array}{l}70.2- \\
81.3\end{array}$ & 761 & 81.4 & $\begin{array}{l}76.8- \\
85.3\end{array}$ & 0.022 \\
\hline $\begin{array}{l}\text { High } \\
\text { risk/harmful } \\
(16-19)\end{array}$ & 209 & 74.9 & $\begin{array}{l}64.5- \\
83.1\end{array}$ & 113 & 68.8 & $\begin{array}{l}55.4- \\
79.7\end{array}$ & 112 & 81.5 & $\begin{array}{l}65.0- \\
91.3\end{array}$ & 0.028 \\
\hline $\begin{array}{l}\text { High } \\
\text { risk/hazardous } \\
(20+)\end{array}$ & 226 & 70.6 & $\begin{array}{l}61.1- \\
78.6\end{array}$ & 135 & 68.7 & $\begin{array}{l}53.1- \\
80.9\end{array}$ & 104 & 72.9 & $\begin{array}{l}58.5- \\
83.7\end{array}$ & 0.480 \\
\hline \multicolumn{11}{|c|}{ Correct HIV knowledge and myth rejection } \\
\hline No knowledge & $\begin{array}{l}12 \\
575\end{array}$ & 74.1 & $\begin{array}{l}72.6- \\
75.5\end{array}$ & $\begin{array}{l}5 \\
760\end{array}$ & 73.0 & $\begin{array}{l}70.9- \\
75.0\end{array}$ & $\begin{array}{l}7 \\
518\end{array}$ & 75.5 & $\begin{array}{l}73.7- \\
77.2\end{array}$ & 0.001 \\
\hline Yes knowledge & $\begin{array}{l}7 \\
337\end{array}$ & 77.1 & $\begin{array}{l}75.3- \\
78.8\end{array}$ & $\begin{array}{l}2 \\
726\end{array}$ & 75.6 & $\begin{array}{l}73.3- \\
77.9\end{array}$ & $\begin{array}{l}5 \\
034\end{array}$ & 78.6 & $\begin{array}{l}76.5^{-} \\
80.6\end{array}$ & 0.003 \\
\hline \multicolumn{11}{|c|}{ Self-perceived risk of HIV infection } \\
\hline No risk & $\begin{array}{l}15 \\
921\end{array}$ & 72.3 & $\begin{array}{l}70.8- \\
73.8\end{array}$ & $\begin{array}{l}6 \\
089\end{array}$ & 69.3 & $\begin{array}{l}67.0- \\
71.4\end{array}$ & $\begin{array}{l}10 \\
681\end{array}$ & 74.8 & $\begin{array}{l}73.2- \\
76.4\end{array}$ & $\begin{array}{l}< \\
0.001\end{array}$ \\
\hline Yes risk & $\begin{array}{l}2 \\
378\end{array}$ & 79.4 & $\begin{array}{l}76.8- \\
81.7\end{array}$ & $\begin{array}{l}1 \\
363\end{array}$ & 78.2 & $\begin{array}{l}74.5- \\
81.5\end{array}$ & $\begin{array}{l}1 \\
186\end{array}$ & 80.8 & $\begin{array}{l}77.5- \\
83.8\end{array}$ & 0.105 \\
\hline \multicolumn{11}{|l|}{$\begin{array}{l}\text { Survey HIV } \\
\text { status }\end{array}$} \\
\hline HIV Positive & $\begin{array}{l}2 \\
358\end{array}$ & 87.6 & $\begin{array}{l}85.1- \\
89.7\end{array}$ & $\begin{array}{l}1 \\
449\end{array}$ & 85.9 & $\begin{array}{l}82.4- \\
88.9\end{array}$ & 909 & 90.0 & $\begin{array}{l}86.6- \\
92.6\end{array}$ & 0.003 \\
\hline HIV Negative & $\begin{array}{l}12 \\
044\end{array}$ & 74.8 & $\begin{array}{l}73.4- \\
76.1\end{array}$ & $\begin{array}{l}4 \\
889\end{array}$ & 72.7 & $\begin{array}{l}70.6- \\
74.8\end{array}$ & $\begin{array}{l}7 \\
155\end{array}$ & 76.2 & $\begin{array}{l}74.6- \\
77.8\end{array}$ & $\begin{array}{l}<.001 \\
0.01\end{array}$ \\
\hline
\end{tabular}

\section{Factors associated with uptake of HIV testing}

Figure 1 shows the final adjusted model for multivariate regression analysis of statistically significant factors independently associated with the uptake of HIV testing by asset-based socio-economic status among respondents aged 15 years and older. Among respondents from low SES households, females were significantly more likely to test for HIV [adjusted odds ratio $(a O R)=3.09(95 \% \mathrm{Cl}: 1.77-5.38), p<0.001$ ] than males. The increased likelihood of HIV testing uptake was significantly associated with respondents with secondary [aOR $=1.61$ (95\% Cl: $1.07-2.41), \mathrm{p}=0.023$ ] and tertiary [aOR $=3.62(95 \% \mathrm{Cl}: 1.24-10.59), \mathrm{p}=0.019$ ] level educational qualifications, compared to those with no education or with primary level education completed. The decreased likelihood of HIV testing uptake was significantly associated with respondents who engaged in low-risk drinking [OR $=0.60(95 \% \mathrm{Cl}: 0.39-0.91), p=0.017]$ and high-risk drinking [OR $=0.27(95 \% \mathrm{Cl}$ : $0.10-0.70), p=0.007]$ compared to those who abstained from alcohol. Respondents who perceived themselves as being at risk of HIV infection were also significantly less likely to test for HIV [OR $=0.66$ ( $95 \% \mathrm{Cl}$ : $0.47-0.94)$, 
$p=0.022]$ compared to their counterparts. The decreased likelihood of HIV testing uptake was significantly associated with respondents who resided in rural formal areas [OR $=0.60(95 \% \mathrm{Cl}: 0.38-0.95), p=0.028$ ] compared to those from urban areas.

Among respondents from high SES households, females were significantly more likely to test for HIV [aOR = 5.23 (95\% Cl: 2.08-13.16), p < 0.001] than males. The decreased likelihood of HIV testing uptake was significantly associated with being White [aOR $=0.28(0.08-0.92), \mathrm{p}=0.036]$, and Indian $/$ Asian $[\mathrm{aOR}=0.12$ (95\% Cl: 0.03-0.39), $p<0.001]$ compared to being Black African. Respondents who were not married were significantly less likely to test for HIV [aOR $=0.03$ (95\% Cl: $0.00-0.27), p<0.001]$ compared to those who reported being married.

\section{Discussion}

This nationally representative population-based study undertaken to identify risk characteristics showed that people with a high socio-economic status reported significantly higher HIV testing uptake than those with a low SES, 76.7\% vs 73.8\%. The differences in HIV testing between high and low SES were found in certain demographic and HIV-related risk characteristics. Bivariate analysis showed that people from high SES that were aged 25 years and older, not married, females, Black African, employed, and residing in all locality types were more likely to have tested for HIV compared to those with a low SES. However, the $90 \%$ target for HIV testing has yet to be achieved. In addition, HIV-related risk characteristics that were significantly associated with HIV testing were self-perceived HIV risk perception, knew survey HIV status result, sexual activity, differences in age of sexual partner, alcohol consumption, and correct knowledge of HIV prevention and transmission.

Findings from the multivariate logistic regression models showed increased uptake of HIV testing was associated with being female and having a higher educational level, independent of SES. Higher levels of HIV testing among females is consistent with global statistics $[22,23]$. The limited male participation in HIV testing observed in our study is worrisome since HIV positive men who are unaware of their status may continue to engage in unsafe behaviour [24]. These results highlight the importance of developing interventions to reach men and address gender disparities in HIV testing.

Our findings are consistent with other studies conducted in sub-Saharan Africa where HIV testing was associated with educational attainment [25-27]. Those with higher educational attainment may be more knowledgeable about HIV and understand the benefits of testing and the importance of knowing one's HIV status and have greater control over the decision to test [28]. Interventions are needed to improve testing rates among the less educated population group.

Similar to other studies, HIV testing uptake was higher in the older age group than among adolescents [29]. Young people report significant psychological barriers to HIV testing including a lack of community support and perceived negative attitudes of health care workers [29,30]. For adolescents in high SES households, addressing their age-specific concerns is important. HIV testing is the first step into both the HIV prevention and treatment cascade, and can positively influence one's sexual risk behaviour and reduce the likelihood of future 
HIV acquisition or further transmission [31]. Addressing this age gap in HIV testing is crucial for improving the awareness of HIV status among young people and to ensure that HIV positive youth receive sustained treatment and are virally suppressed to save lives and curtail the transmission of HIV.

In agreement with other studies, our findings showed that uptake of HIV testing in the rural informal areas was low compared to the urban areas [32]. This demonstrates that this rural-urban gap in testing persists in the modern era of effective HIV therapy when early diagnosis and linkage to care as part of the UNAIDS 90-90-90 targets is even more essential. Some overarching factors include poverty in many rural areas, limited resources, and structural barriers that pose challenges to accessing HIV testing services [33]. This accentuates the need for scaling up effective models for delivering HIV testing in remote areas, such as mobile and home-based testing [34]. Provider-initiated modes of testing may also be more accessible to lower socio-economic groups than traditional HIV counselling and testing. Testing through the prevention of mother-to-child transmission services has proven to minimise the socio-economic hurdles faced [8]. Context-based strategies are therefore crucial to improve access to HIV testing services [4, 14], and cognisance needs to be taken of those who do not interact with the health system often [15].

Consistent with current findings, other studies also showed that married women were more likely to undergo HIV testing than unmarried women $[35,36]$. This may be reflective of marriage as a supportive relationship that motivates couples to seek HIV testing [36]. Couples are also more likely to undergo HIV testing when they are planning their marriage to know their HIV serostatus, than those who have never been married [35]. Targeted interventions are needed to increase HIV testing among those that are not married.

The results showed that Whites and Indians/Asians were less likely to test for HIV compared to Black Africans who form the majority in the South African context. This is because of the underlying evidence-based narrative that suggests Black Africans are at greater risk of HIV infection for historical reasons, which account for both the economic inequality and the poverty that pervade this racial group. Elsewhere, racial differences in HIV testing have been attributed to the health services utilization model [37]. HIV testing was associated generally with the self-perceived risk of HIV among minority groups. There is a need to improve current practices to reduce health disparities generally, and disparities in HIV testing in particular. [37]. In line with other studies, the findings also revealed that not being married was associated with low uptake of HIV testing [25, 35]. This is worrying given that not knowing one's HIV status is a major impediment to accessing appropriate HIV prevention, care, and treatment [38]. Individuals in non-marital relationships may be more fearful of HIV positive test results. This raises serious public health concerns when considering that the majority of these individuals may be engaging in unprotected sex and would benefit from being aware of their HIV status [38]. There is a need to devise alternative approaches to create demand for the uptake of HIV testing services among nonmarried individuals with no prior exposure to HIV testing.

Low and high risk alcohol drinking was associated with low HIV testing uptake, independent of SES. Elsewhere, alcohol consumption has also been observed as a barrier to prior HIV testing in a population-based study [39]. Alcohol use has been consistently associated with risky sexual behaviour [40,41]. This highlights the importance of addressing social risk behaviours such as alcohol consumption, towards improving uptake of HIV testing and the prevention of HIV transmission. 
High self-perceived risk of HIV infection was associated with low uptake of HIV testing. This implies that individuals might refuse HIV testing even if they know that they are at high risk of HIV infection. This differs from the view that people who refuse HIV testing commonly do so because they do not perceive themselves to be at risk [42]. Either way self-perceived risk is an important factor in the uptake of HIV testing. This emphasizes a need to assess whether an individual's self-perception of their HIV risk is accurate - to encourage testing, make the diagnosis, and link patients to care and treatment services [43].

The study has some limitations. Due to the cross-sectional design of the current study, causal inferences cannot be drawn, and the analysis can only identify associations. Additionally, the strength of the association was limited by wide confidence intervals in some instances. Furthermore, the analysis is based on self-report of HIV testing and risk factors, which are prone to social desirability and recall bias. Despite these limitations, this study used a nationally representative sample, and the findings are generalizable to the entire country.

\section{Conclusions}

The findings from this study show that socio-demographic factors such as gender, race, locality, and including socio-behavioural factors such as alcohol consumption and self-perceived risk of HIV infection, have different outcomes for HIV testing. Interventions to improve HIV testing should target these barriers to increase HIV testing uptake. Reaching the first 90 of the $90-90-90$ targets will require efforts to increase the uptake of HIV testing by addressing the issues, among others, HIV risk perception, accessibility of services, community knowledge, and awareness.

\section{List Of Abbreviations}

AIDS Acquired Immune Deficiency Syndrome

ART Antiretroviral therapy

Cl Confidence Interval

HIV Human Immunodeficiency Virus

MCA Multiple correspondence analysis

SAL Small area layer

SES Socioeconomic status

SSA Sub-Saharan Africa

UNAIDS Joint United Nations Programme on HIV/AIDS

VP Visiting Point

\section{Declarations}




\section{Ethics approval and consent to participate}

The survey protocol was approved by the HSRC Research Ethics Committee (REC: 4/18/11/15), and the Associate Director for Science, Center for Global Health, Centers for Disease Control and Prevention (CDC)). Ethical clearance was also obtained from the University of KwaZulu-Natal's Biomedical Research Ethics Committee (BE 646/18). Informed consent was obtained before undertaking both the behavioural data and specimen collection.

\section{Consent for publication}

Not applicable

\section{Availability of data and materials}

The datasets used and/or analysed during the current study are available from the corresponding author on reasonable request.

\section{Competing interests}

The authors declare that they have no competing interests

\section{Funding}

This manuscript was supported by the President's Emergency Plan for AIDS Relief (PEPFAR) through the Centers for Disease Control and Prevention, under the terms of Cooperative Agreement Number [NU2GGH001629]. The findings and conclusions in this report are those of the author(s) and do not necessarily represent the official position of the funding agencies.

\section{Authors' contributions}

SJ drafted of the manuscript. SJ and MM performed the statistical analysis. SJ, MM, AN, YS, MT and LS participated in the implementation of the survey that provided the data for the manuscript. All authors contributed to the review of draft manuscript and approved the final manuscript.

\section{Acknowledgements}

Not applicable

\section{Authors' information (optional)}




\section{References}

1. UNAIDS. Miles to Go. Global AIDS Update. Geneva: Joint United Nations Programme on HIV/AIDS; 2018.

2. Simbayi LC, Zuma K, Zungu N, Moyo S, Marinda E, Jooste S, et al. South African national HIV prevalence, incidence, behaviour and communication survey, 2017. Cape Town: HSRC Press; 2019.

3. UNAIDS. The Gap Report. Geneva: Joint United Nations Programme on HIV/AIDS: Joint United Nations Programme on HIV/AIDS; 2014.

4. Peltzer K, Matseke G, Mzolo T, Majaja M. Determinants of knowledge of HIV status in South Africa: results from a population-based HIV survey. BMC Public Health. 2009;9(1):174.

5. Musheke M, Ntalasha H, Gari S, McKenzie O, Bond V, Martin-Hilber A, et al. A systematic review of qualitative findings on factors enabling and deterring uptake of HIV testing in Sub-Saharan Africa. BMC Public Health. 2013;13(1):220.

6. Chemaitelly H, Cremin I, Shelton J, Hallett TB, Abu-Raddad LJ. Distinct HIV discordancy patterns by epidemic size in stable sexual partnerships in sub-Saharan Africa. Sexually Transmitted Infections. 2012;88(1):51.

7. Jean K, Anglaret X, Moh R, Lert F, Dray-Spira R. Barriers to HIV Testing in Côte d'Ivoire: The Role of Individual Characteristics and Testing Modalities. PLOS ONE. 2012;7(7):e41353.

8. Obermeyer CM, Neuman M, Hardon A, Desclaux A, Wanyenze R, Ky-Zerbo O, et al. Socio-economic determinants of HIV testing and counselling: a comparative study in four African countries. Tropical Medicine \& International Health. 2013;18(9):1110-8.

9. Deblonde J, De Koker P, Hamers FF, Fontaine J, Luchters S, Temmerman M. Barriers to HIV testing in Europe: a systematic review. European Journal of Public Health. 2010;20(4):422-32.

10. Mohlabane N, Tutshana B, Peltzer K, Mwisongo A. Barriers and facilitators associated with HIV testing uptake in South African health facilities offering HIV Counselling and Testing. Health SA Gesondheid. 2016;21:86-95.

11. Nuwaha F, Kabatesi D, Muganwa M, Whalen CC. Factors influencing acceptability of voluntary counselling and testing for HIV in Bushenyi district of Uganda. East Afr Med J. 2002;79(12):626-32.

12. Wringe A, Isingo R, Urassa M, Maiseli G, Manyalla R, Changalucha J, et al. Uptake of HIV voluntary counselling and testing services in rural Tanzania: Implications for effective HIV prevention and equitable access to treatment. Tropical medicine \& international health : TM \& IH. 2008;13:319-27.

13. Maman S, van Rooyen H, Groves AK. HIV status disclosure to families for social support in South Africa (NIMH Project Accept/HPTN 043). AIDS Care. 2014;26(2):226-32.

14. Teklehaimanot HD, Teklehaimanot A, Yohannes M, Biratu D. Factors influencing the uptake of voluntary HIV counseling and testing in rural Ethiopia: a cross sectional study. BMC Public Health. 2016;16(1):239.

15. Mitchell S, Cockcroft A, Lamothe G, Andersson N. Equity in HIV testing: evidence from a cross-sectional study in ten Southern African countries. BMC International Health and Human Rights. 2010;10(1):23.

16. Wabiri N, Taffa N. Socio-economic inequality and HIV in South Africa. BMC Public Health. 2013;13(1):1037.

17. StatsSA. Mid-year population estimates 2017. Pretoria: Statistics South Africa; 2017. 
18. Berg S, Burger R, von Maltitz M, Rand G. Using an Asset Index to Assess Trends in Poverty in Seven SubSaharan African Countries. World Development. 2008;36:1113-30.

19. Saunders JB, Aasland OG, Babor TF, De La Fuente JR, Grant M. Development of the Alcohol Use Disorders Identification Test (AUDIT): WHO Collaborative Project on Early Detection of Persons with Harmful Alcohol Consumption-II. Addiction. 1993;88(6):791-804.

20. Morojele NK, Nkosi S, Kekwaletswe CT, Shuper PA, Manda SO, Myers B, et al. Utility of Brief Versions of the Alcohol Use Disorders Identification Test (AUDIT) to Identify Excessive Drinking Among Patients in HIV Care in South Africa. Journal of Studies on Alcohol and Drugs. 2016;78(1):88-96.

21. Jann B. Plotting Regression Coefficients and other Estimates. The Stata Journal. 2014;14(4):708-37.

22. WHO. Guideline on when to start antiretroviral therapy and on pre-exposure prophylaxis for HIV. Geneva: World Health Organization: World Health Organization; 2015.

23. WHO. Global tuberculosis report 2019. Geneva: World Health Organization: World Health Organization; 2019.

24. Mills EJ, Beyrer C, Birungi J, Dybul MR. Engaging Men in Prevention and Care for HIV/AIDS in Africa. PLOS Medicine. 2012;9(2):e1001167.

25. Tenkorang EY, Owusu GA. Correlates of HIV testing among women in Ghana: some evidence from the Demographic and Health Surveys. AIDS Care. 2010;22(3):296-307.

26. Hutchinson PL, Mahlalela X. Utilization of voluntary counseling and testing services in the Eastern Cape, South Africa. Taylor \& Francis; 2006 2006/07/01. Report No.: 0954-0121 Contract No.: 5.

27. Venkatesh KK, Madiba P, De Bruyn G, Lurie MN, Coates TJ, Gray GE. Who gets tested for HIV in a South African urban township? Implications for test and treat and gender-based prevention interventions. J Acquir Immune Defic Syndr. 2011;56(2):151-65.

28. Jukes M, Simmons S, Bundy D. Education and vulnerability: the role of schools in protecting young women and girls from HIV in southern Africa. AIDS. 2008;22.

29. Zanoni BC, Elliott RJ, Neilan AM, Haberer JE. Screening for HIV and linkage to care in adolescents: insights from a systematic review of recent interventions in high- versus low- and middle-income settings. Adolesc Health Med Ther. 2018;9:211-35.

30. MacPhail CL, Pettifor A, Coates T, Rees H. "You Must Do the Test to Know Your Status": Attitudes to HIV Voluntary Counseling and Testing for Adolescents Among South African Youth and Parents. Health Education \& Behavior. 2006;35(1):87-104.

31. Fonner VA, Denison J, Kennedy CE, O'Reilly K, Sweat M. Voluntary counseling and testing (VCT) for changing HIV-related risk behavior in developing countries. Cochrane Database of Systematic Reviews. 2012(9).

32. CSA., ICF. Ethiopia Demographic and Health Survey 2011. Addis Ababa, Ethiopia and Calverton, Maryland, USA.: Central Statistical Agency and ICF International.; 2012.

33. Mwangi M, Kellogg TA, Dadabhai SS, Bunnell R, Baltazar G, Ngare C, et al. Factors Associated with Uptake of HIV Test Results in a Nationally Representative Population-Based AIDS Indicator Survey. Open AIDS J. 2014;8:7-16.

34. Maheswaran H, Thulare H, Stanistreet D, Tanser F, Newell M-L. Starting a home and mobile HIV testing service in a rural area of South Africa. J Acquir Immune Defic Syndr. 2012;59(3):e43-e6. 
35. Ziraba AK, Madise NJ, Kimani JK, Oti S, Mgomella G, Matilu M, et al. Determinants for HIV testing and counselling in Nairobi urban informal settlements. BMC public health. 2011;11:663-.

36. Chirwa E, Malata A, Norr K. HIV prevention awareness and practices among married couples in Malawi. Malawi medical journal : the journal of Medical Association of Malawi. 2011;23:32-7.

37. Lo CC, Runnels RC, Cheng TC. Racial/ethnic differences in HIV testing: An application of the health services utilization model. SAGE Open Medicine. 2018;6:2050312118783414.

38. Cherutich P, Kaiser R, Galbraith J, Williamson J, Shiraishi RW, Ngare C, et al. Lack of Knowledge of HIV Status a Major Barrier to HIV Prevention, Care and Treatment Efforts in Kenya: Results from a Nationally Representative Study. PLOS ONE. 2012;7(5):e36797.

39. Fatch R, Bellows B, Bagenda F, Mulogo E, Weiser S, Hahn JA. Alcohol consumption as a barrier to prior HIV testing in a population-based study in rural Uganda. AIDS Behav. 2013;17(5):1713-23.

40. Kalichman SC, Simbayi LC, Kaufman M, Cain D, Jooste S. Alcohol Use and Sexual Risks for HIV/AIDS in Sub-Saharan Africa: Systematic Review of Empirical Findings. Prevention Science. 2007;8(2):141.

41. Woolf-King SE, Maisto SA. Alcohol Use and High-Risk Sexual Behavior in Sub-Saharan Africa: A Narrative Review. Archives of Sexual Behavior. 2011;40(1):17-42.

42. Takahashi TA, Johnson KM, Bradley KA. A population-based study of HIV testing practices and perceptions in 4 U.S. states. J Gen Intern Med. 2005;20(7):618-22.

43. Pringle K, Merchant RC, Clark MA. Is self-perceived HIV risk congruent with reported HIV risk among traditionally lower HIV risk and prevalence adult emergency department patients? Implications for HIV testing. AIDS Patient Care STDS. 2013;27(10):573-84.

\section{Figures}




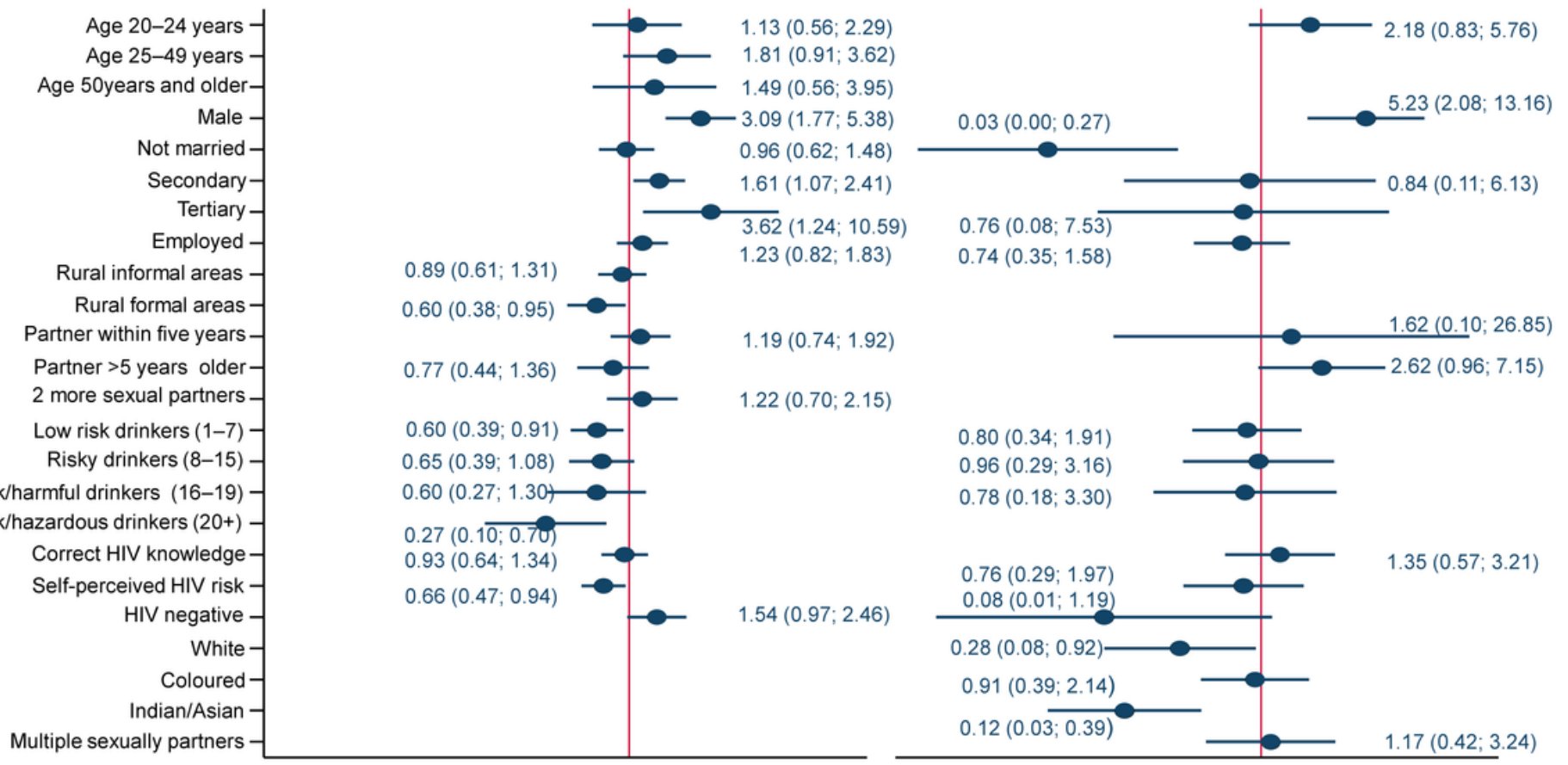

Adjusted odds ratio

\section{Figure 1}

Factors associated with HIV testing by SES among 15 years and older, South Africa 2017

Low socio-economic status

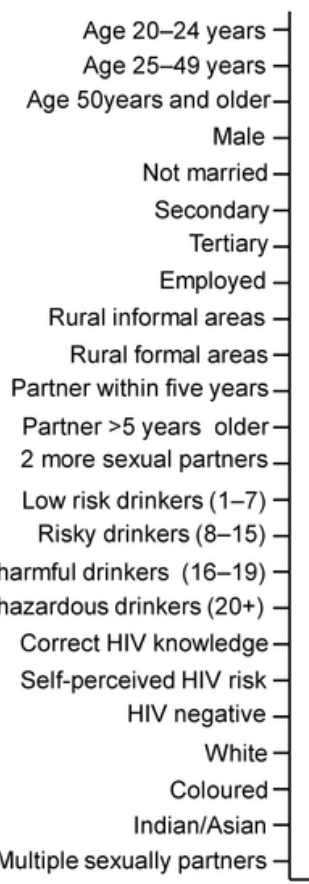

High socio-economic status

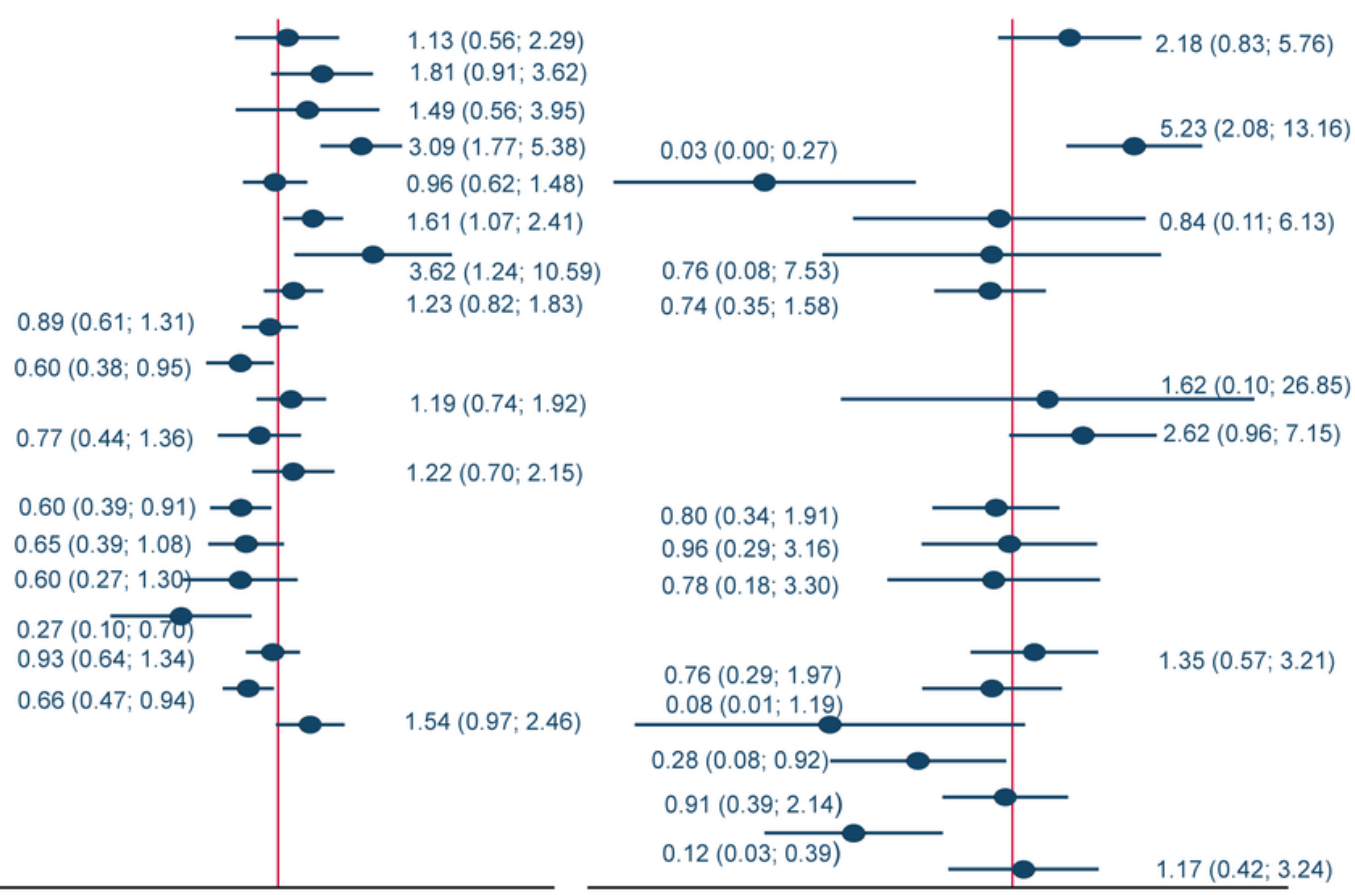

Adjusted odds ratio

Figure 1 
Factors associated with HIV testing by SES among 15 years and older, South Africa 2017

Page 20/20 